\title{
Poster: Quaternion Based General Purpose Techniques for Activity Recognition Using Wearables
}

\author{
Md Abu Sayeed Mondol \\ University of Virginia \\ Charlottesville, VA, USA \\ mondol@virginia.edu \\ John A. Stankovic \\ University of Virginia \\ Charlottesville, VA, USA \\ stankovic@virginia.edu
}

Permission to make digital or hard copies of part or all of this work for personal or classroom use is granted without fee provided that copies are not made or distributed for profit or commercial advantage and that copies bear this notice and the full citation on the first page. Copyrights for third-party components of this work must be honored. For all other uses, contact the owner/author(s).

Copyright held by the owner/author(s)

UbiComp/ISWC'18 Adjunct, October 8-12, 2018, Singapore, Singapore ACM 978-1-4503-5966-5/18/10.

https://doi.org/10.1145/3267305.326766

\begin{abstract}
Human activity recognition (HAR) is challenging, particularly in natural settings, due to issues like confounding gestures present in different activities, diversity in performing the same activity, and the wide range of possible human activities. Acceleration and rotation rate, two of the most widely used sensing modalities for HAR, are limited in addressing these issues. Also, many solutions for wearables are focused on some particular activities, and they do not generalize to others. One challenge is to develop underlying generic techniques for activity recognition that can be used in many different wearable based applications. We present a set of general purpose techniques for activity recognition using wearables. The techniques are based on quaternions that represent the orientation of a device in three-dimensional space. The techniques can be used for different purposes like reducing computation, increasing robustness and accuracy, and better understanding movements for HAR.
\end{abstract}

\section{Author Keywords}

Activity Recognition; Wearable Sensor; Quaternion

\section{ACM Classification Keywords}

[Human-centered computing; Ubiquitous and mobile computing; Ubiquitous and mobile computing theory, concepts and paradigms] 


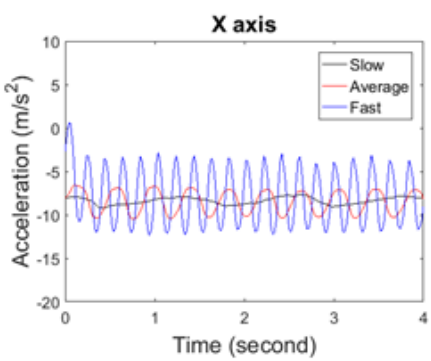

(a)

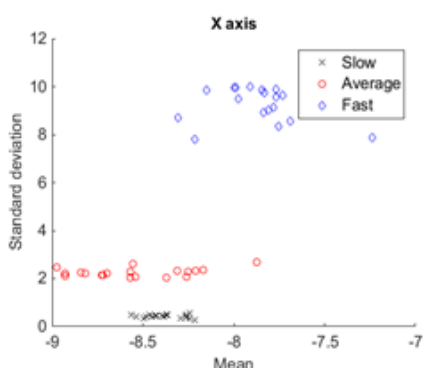

(b)

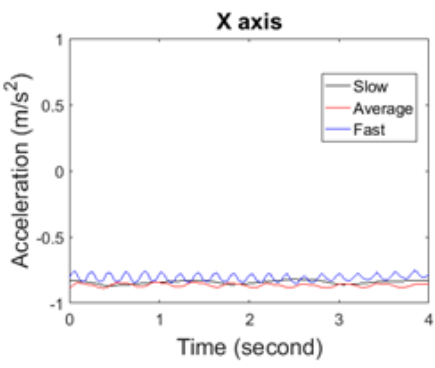

(c)

Figure 1: For brushing teeth at different intensity (a) Accelerations along the $X$ axis, (b) Mean and standard deviation from 1 second segments, (c) Gravity vectors along the $X$ axis.

\section{Introduction}

The accelerometer and the gyroscope are the most widely used wearable inertial sensors for HAR. However, they are not, in general, robust enough in recognizing the wide range of human activities involving complex and interleaving body gestures. For example, Figure 1(a) shows the acceleration values along the $X$ axis from a wrist-worn accelerometer for brushing teeth in a specific way, but at different speeds (slow, average, fast). As shown in the figure, the data differs significantly for the different speeds even though the gestures are nearly the same. Similar variations are also present in the data from the $Y$ and the $Z$ axes. Dissimilarity in the sensor data results in dissimilarity in the features for the same activity, consequently results more similarity among features from different classes. Figure 1 (b) shows two widely used features, mean and standard deviation, extracted from 1-second segments from the acceleration data along the $X$ axis. It demonstrates significant variance in the feature values even though they are from the similar gestures. Similar to the accelerometers, the gyroscopes also suffer from these problems.

Today, many solutions for wearables are focused on some particular activities, and they do not generalize to others.

One challenge is to develop underlying generic techniques that can be used in many different wearable based applications, and that would support in addressing several challenges associated with HAR. We present a set of general purpose techniques for activity recognition using wearables. The techniques are based on quaternions that represent the orientation of an object in three-dimensional space. Quaternions are widely used in different areas including computer graphics, gaming, robotics, and computer vision. There do exist some works for HAR that use quaternions. However, these methods are designed and developed to recognize specific activities [2]. In contrast, our techniques are not activity specific, and they can be used to develop better solutions for HAR. However, they are not a complete replacement of the existing methods, and can be used in combination with the state of the art techniques.

\section{General Purpose Techniques}

1. Orientation Decomposition

As mentioned earlier, our techniques are based on the quaternions that represent the orientation of a coordinate system with respect to another coordinate system. In the context of smart devices like smart phones and smart watches, a quaternion represents the orientation of the device with respect to the world coordinate system. A quaternion $q$ is formally defined by a scalar component $\left(q_{s}\right)$ and a 3D vector $\left(q_{x}, q_{y}, q_{z}\right)$ as $q=q_{s}+q_{x} \hat{i}+q_{y} \hat{j}+q_{z} \hat{k}$, where $i, j$, and $k$ are the imaginary basis elements. The quaternion is called a unit quaternion if its magnitude equals to one, i.e. if $|q|=\sqrt{q_{s}^{2}+q_{x}^{2}+q_{y}^{2}+q_{z}^{2}}=1$. The quaternions are usually computed through fusing the outputs of multiple sensors such as an accelerometer, a gyroscope, and a magnetometer. Many of the commercially off-the-shelf devices like the smart phones and the smart watches provide the unit quaternions

The quaternion values depend on the orientation of the device with respect to both the earth gravity and the direction of the earth magnetic field (e.g., north or south). Such dependency of the quaternion on direction limits its usage for HAR where a user can perform an activity facing in any direction. We use rotation matrix, an equivalent representation of the quaternion, to decompose the orientation. A rotation matrix is defined as: 


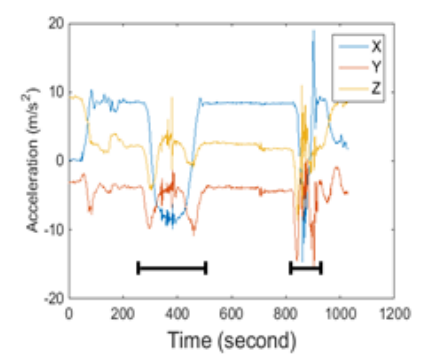

(a)

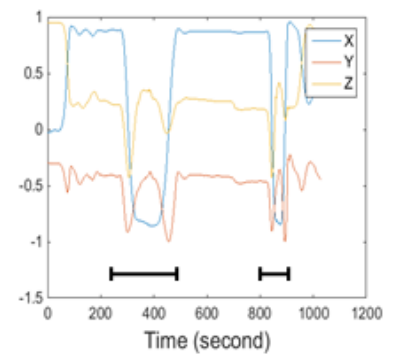

(b)

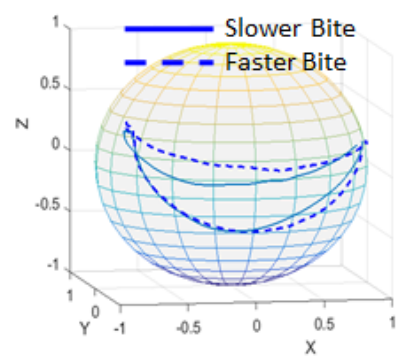

(c)

Figure 2: For two similar apple bites at different speeds plot of (a) The accelerations and (b) The gravity vectors along the time axis,

(c) The spatiotemporal representation of the gravity vectors.

$$
\left[\begin{array}{ccc}
q_{s}^{2}+q_{x}^{2}-q_{y}^{2}-q_{z}^{2} & 2\left(q_{x} q_{y}-q_{s} q_{z}\right) & 2\left(q_{x} q_{z}+q_{s} q_{y}\right) \\
2\left(q_{s} q_{z}+q_{x} q_{2}\right) & q_{s}^{2}-q_{x}^{2}+q_{y}^{2}-q_{z}^{2} & 2\left(q_{x} q_{z}+q_{s} q_{y}\right) \\
2\left(q_{s} q_{z}-q_{s} q_{2}\right) & 2\left(q_{y} q_{z}+q_{s} q_{x}\right) & q_{s}^{2}-q_{x}^{2}-q_{y}^{2}+q_{z}^{2}
\end{array}\right]
$$

The rows and columns of the matrix are orthonormal (orthogonal unit vectors). The first, second and third rows represent orientation of the device with respect to east, north and gravity of the world, respectively. So, only the gravity vectors are used for direction agnostic activity recognition tasks. The other two vectors are used where direction is relevant.

It should be noted that the acceleration values are the combination of the earth gravity and the linear accelerations due to movement. When there is no movement, the magnitudes of the accelerations equal to the earth gravity $\left(\approx 9.8 \mathrm{~ms}^{-2}\right)$, and normalizing the accelerations results the unit gravity vectors. However, movements from activity adds linear accelerations that depend on the intensity of the movements even for the same gestures (Figure 1 (a)). On the other hand, the gravity vectors are less distorted as shown in Figure 1 (c). In contrast to the accelerometers, the gyroscopes and the magnetometers, the unit vectors from the rotation matrix are less distorted for the same gestures, providing more consistent features for the same activity.

\section{Spatiotemporal Representation}

Since the vectors are unit in length, they can be placed on the surface of a unit sphere. In contrast to the time domain representation where the vectors are plotted against time, the spatiotemporal representation is characterized by the locations of the unit vectors on the sphere and by the order of the unit vectors over time. For example, Figure 2(a) and Figure 2(b) show the acceleration and unit gravity data from the right wrist, respectively, for two similar apple bites.
Figure 2(c) shows the spatiotemporal representation of the bites. Here, the later one shows more similarity between the bites than the others. Such representation not only provides useful insights about the gestures, but also can be used to address temporal variations in the gestures.

\section{Orientation Reachability}

We define the Orientation Reachability for an activity by the area on the sphere that is covered by the unit vectors of that activity. For example, Figure 3(a) shows the orientation reachability of the gravity vectors for brushing teeth activity. The area is very small compared to the orientation reachability of all possible activities of daily living. This property can be used to separate most of the data from other activities before additional feature extraction or processing. This early pruning results in less computation on average for recognizing the activities of interest.

In many contexts, for example in homes and offices, information about the direction of a user's body or body parts helps in better recognizing different activities of the user. For example, Figure 3(b) and 3(c) show the unit gravity vectors and the unit north vectors from a wrist device, respectively, for opening two different doors that are similar, but facing two different directions. As shown, the doors can be easily distinguished by the location of the unit north vectors on the sphere. On the other hand, the unit gravity vectors are not suitable in distinguishing the doors, but it is useful to differentiate from other activities. So, the orientation reachability for the north or east vectors are used in combination with that of the gravity vectors where direction provides useful information. In addition to better activity recognition, orientation reachability can also be used to find abnormality in the data. 


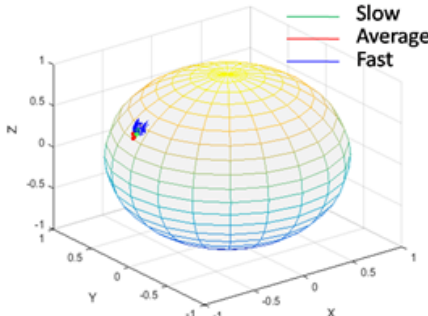

(a)

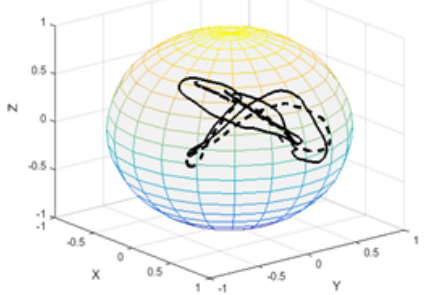

(b)

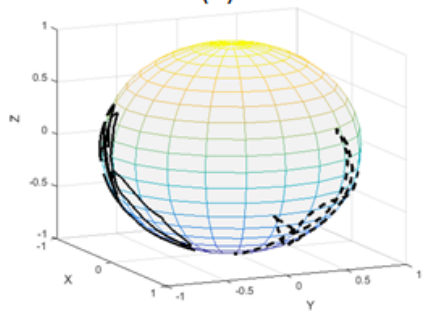

(c)

Figure 3: Orientation Reachability for (a) the gravity vectors for brushing teeth, (b) the gravity vectors for opening two doors, (c) the north vectors for opening the doors.

\section{Sphere Segmentation}

For the better understanding and visualization of the orientation traces, the unit sphere is divided into some nearly uniform cells as shown in Figure 4. The cells on the unit sphere are generated by the Voronoi diagram generated from a regular icosahedron. Fine grained cells are generated by dividing the icosahedron repeatedly. Each of the cells is annotated with a unique number that is useful in understanding the orientation trace or reachability for different activities. The numbers are not shown here due to smaller image size influenced by the space limitation. Icosahedron based division of the sphere surface is widely used in areas like meteorology.
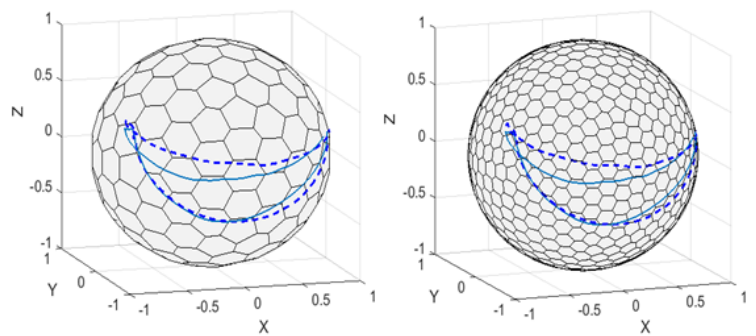

Figure 4: Segmenting the unit sphere

\section{Discussion}

We have done some preliminary studies on the techniques using a public dataset [1]. Results indicate that the techniques are promising for different aspect of HAR including increasing robustness and accuracy, reducing computation requirements, and better visualization. For example, Figure 5 shows the orientation reachability of the right wrist for some eating bites when the wrist is moved to the mouth. We can see the bite moments are clustered in some specific areas of the sphere. The techniques are not activity

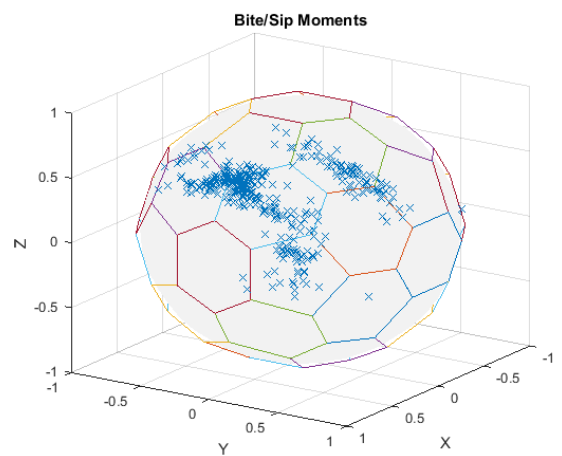

Figure 5: Orientation Reachability of some eating bites

specific, and they have the potential to be used for wide range of activity recognition and monitoring tasks.

\section{Acknowledgements}

This work was supported, in part, by NSF CNS-1646470 and NSF CSR-1527540.

\section{REFERENCES}

1. Ricardo Chavarriaga, Hesam Sagha, Alberto Calatroni, Sundara Tejaswi Digumarti, Gerhard Tröster, José del R Millán, and Daniel Roggen. 2013. The

Opportunity challenge: A benchmark database for on-body sensor-based activity recognition. Pattern Recognition Letters 34, 15 (2013), 2033-2042.

2. Abhinav Parate, Meng-Chieh Chiu, Chaniel Chadowitz, Deepak Ganesan, and Evangelos Kalogerakis. 2014. Risq: Recognizing smoking gestures with inertial sensors on a wristband. In Proceedings of the 12th annual international conference on Mobile systems, applications, and services. ACM, 149-161. 